\title{
Tourniquets and Adductor Canal Blocks in Total Knee Arthroplasty: Is There a Synergistic Effect on Postoperative Pain and Quadriceps Weakness?
}

\author{
Macy M. Dimmett, ${ }^{1}$ Mary Ziemba-Davis, ${ }^{2}$ \\ Leonard T. Buller, ${ }^{2}$ R. Michael Meneghini ${ }^{2}$ \\ ${ }^{1}$ Indiana University School of Medicine \\ ${ }^{2}$ Indiana University School of Medicine, Department of Orthopaedic Surgery \\ Background and Hypothesis: Adequate pain control and avoidance of falls are crucial for \\ successful early recovery following total knee arthroplasty (TKA). Studies have shown an \\ increase in postoperative quadriceps weakness $(\mathrm{QW})$ and pain associated with tourniquet use \\ during TKA. Additionally, increasing doses of adductor canal block (ACB) anesthetic per unit of \\ $\mathrm{BMI}$ are associated with QW after TKA. This retrospective cohort study examined whether \\ tourniquets and ACBs have a synergistic effect on postoperative $\mathrm{QW}$ and pain.
}

Experimental Design or Project Methods: 348 TKAs in 319 patients performed by a single surgeon between October 2015 and May 2018 were retrospectively reviewed. Outcomes included QW as documented in the physical therapy evaluation, average pain score during the first 24 hours after PACU discharge, average pain score during the remainder of the hospital stay, and final pain score prior to discharge.

Results: Sex, age, and BMI did not differ in patients with ACBs with $(n=177)$ and without $(n=168)$ tourniquet use $(p \geq 0.330)$. The overall prevalence of $Q W$ in the sample was $8.7 \%$. Proportionally more patients with ACBs and tourniquets (11.6\%) had $\mathrm{QW}$ compared to patients with $\mathrm{ACBs}$ without tourniquets $(6.1 \%)$, but the difference was not statistically significant with the numbers available. Patient-reported pain scores at the three time points did not differ significantly between patients who received ACBs with or without tourniquets.

Conclusion and Potential Impact: There appears to be no synergistic effect of ACBs and tourniquet use on postoperative pain. However, due to the effect size and number of cases in each group, the synergistic effect of ACBs and tourniquet use on QW deserves additional study with a larger sample size as these results may impact functional recovery and postoperative complications among patients undergoing primary TKA. 